\title{
Crises politiques, crises et anti-crises rhétoriques
}

Political crises, rhetorical crises and anti-crises

\section{Alban Bouvier}

\section{OpenEdition}

\section{Journals}

Édition électronique

URL : http://journals.openedition.org/questionsdecommunication/2319

DOI : 10.4000/questionsdecommunication.2319

ISSN : 2259-8901

\section{Éditeur}

Presses universitaires de Lorraine

\section{Édition imprimée}

Date de publication : 31 décembre 2007

Pagination : 89-102

ISBN : $978-2-86480-849-7$

ISSN : 1633-5961

\section{Référence électronique}

Alban Bouvier, "Crises politiques, crises et anti-crises rhétoriques », Questions de communication [En ligne], 12 | 2007, mis en ligne le 06 avril 2012, consulté le 08 avril 2021. URL : http:// journals.openedition.org/questionsdecommunication/2319; DOI : https://doi.org/10.4000/ questionsdecommunication.2319 


\title{
$>$ DOSSIER
}

\author{
ALBAN BOUVIER \\ Institut Jean Nicod, CNRS, EHESS, ENS, Paris \\ Université de Provence Aix-Marseille 1 \\ bouvier.alban@wanadoo.fr
}

\section{CRISES POLITIQUES, CRISES ET ANTI-CRISES RHÉTORIQUES}

\begin{abstract}
Résumé. - L'auteur prend acte de l'actuelle crise politique aiguë de légitimité des démocraties représentatives. Cependant, il nie qu'elle soit concomitante d'une crise rhétorique si l'on entend par là une valorisation de la seule «mauvaise rhétorique », à savoir des moyens de persuader efficaces, mais logiquement ou éthiquement suspects. Tout au contraire, il prétend qu'on assiste à une valorisation (ou une revalorisation) des débats politiques argumentés, précisément destinés à sortir de la crise politique en question, de sorte qu'il faudrait plutôt parler d'une anti-crise rhétorique. Cette valorisation de la saine argumentation s'observe à différents niveaux. II convient de distinguer ceux-ci (démocratie de proximité, débats parlementaires), tandis que la notion équivoque de démocratie délibérative tend malheureusement à les confondre.
\end{abstract}

Mots clés. - Argumentation, rhétorique, démocratie délibérative, démocratie de proximité, démocratie parlementaire, légitimité politique. 
I e commencerai par distinguer argumentation et rhétorique, sans prétendre que cela correspond à tous les usages de ces termes dont le sens est relativement indéterminé, mais en avançant néanmoins que cela ne s'écarte guère des usages dominants. J'entendrai par « discours argumentatif » une procédure discursive qui vise à persuader rationnellement en trois sens du terme «rationnellement » : celle-ci veut respecter les règles de la logique, celles de l'éthique, et enfin, elle veut être efficacel. Je considérerai comme rhétorique une procédure discursive qui poursuit l'objectif de persuader rationnellement, mais selon un sens unique du terme « rationnellement »: elle ambitionne la seule efficacité de la procédure de persuasion, ce qui implique qu'elle peut faire des entorses à la logique et à l'éthique si cela s'avère le moyen d'une plus grande efficacité. En conséquence, je reprendrai volontiers la distinction de Platon entre une «bonne » et une «mauvaise » rhétorique au sens où la bonne rhétorique recherche l'efficacité tout en respectant les normes de la logique et de l'éthique, à la différence de la mauvaise qui ne recherche que l'efficacité 2 . Au sens où je prends ces termes, l'argumentation requiert donc la rhétorique, mais ne s'y réduit pas. Enfin, j'entendrai par crise rhétorique non pas une crise de la rhétorique au sens où, dans la période considérée, on douterait de la pertinence d'utiliser des moyens discursifs réputés efficaces pour persuader et, a fortiori, de la pertinence d'enseigner la rhétorique ${ }^{3}$, mais au contraire une période critique pendant laquelle la rhétorique (au sens du seul art de persuader par des moyens efficaces, quoi qu'il en soit de la validité logique et éthique du raisonnement) est valorisée au détriment de l'argumentation dans un secteur où un autre de l'existence. La crise rhétorique est donc une crise de la reconnaissance de la valeur de l'argumentation, c'est-à-dire une mise en cause de celle-ci comme procédure de persuasion et une valorisation de la seule « mauvaise rhétorique $»^{4}$.

\footnotetext{
' Pour ne pas multiplier les termes, j'utilise ici « logique » en un sens large et ancien qui était encore celui de J. S. Mill ( 1843 ) et qui inclut non seulement les règles de la logique formelle mais aussi l'accord avec le réel ; ainsi quelque chose qui est empiriquement faux sera-t-il dit transgresser les règles de la « logique » en ce sens là. J. S. Mill aurait parlé de « sophisme de simple inspection » et de « sophismes d'observation ».

2 Bien entendu, les procédures effectives de l'une et l'autre rhétoriques peuvent être ellesmêmes plus ou moins efficaces et donc la bonne comme la mauvaise rhétorique plus ou moins réussies.

3 Sur ce point, voir Fr. Douay (2007).

${ }^{4}$ II ne s'agit donc pas de crise de la rhétorique au sens où la recherche des moyens efficaces de convaincre serait en tant que telle condamnée ou dévalorisée, soit que l'on réduise la rhétorique à la seule mauvaise rhétorique soit que l'on prétende que les discours logiquement ou éthiquement valides sont d'eux-mêmes efficaces sans qu'il soit besoin de recourir à des procédures spéciales. On rencontre de telles crises au temps de Platon et également à celui de Descartes.
} 
En conséquence, je verrai dans la valorisation ou revalorisation du recours à l'argumentation au sens ici défini (qui est en quelque sorte un sens plein du terme) l'antidote naturel des crises rhétoriques (au sens défini). II conviendrait donc de parler plutôt d'anti-crise rhétorique pour caractériser des périodes où l'argumentation (au sens défini) et donc la « bonne rhétorique » sont valorisées. Je distinguerai donc soigneusement le rôle que peuvent jouer rhétorique et argumentation dans la vie politique contemporaine, mon but étant, dans cet article, de circonscrire un certain nombre de cas de figure distincts. Je laisserai de côté ici parce que cela demanderait un traitement spécifique - ce qui me semble une authentique crise rhétorique au sens défini, et, en l'occurrence, une crise particulièrement tragique de l'histoire des démocraties occidentales contemporaines, celle de l'entre-deux guerres et, plus spécialement celle de la République de Weimar, crise qui est concomitante de la montée de l'hitlérisme et des fascismes ou, plus exactement, des populismes ${ }^{5}$.

Je me concentrerai sur un examen approfondi de ce que, au contraire, on peut considérer comme étant une anti-crise rhétorique puisque c'est ce qui sera ici en question, c'est la valorisation ou la revalorisation de la valeur de l'argumentation comme forme de réponse à une crise politique ou, peutêtre, à différents types de crises politiques. Cette anti-crise présente ellemême deux aspects bien distincts. Le premier, très visible dans certains pays occidentaux, correspond à l'émergence d'une nouvelle conception de l'autorité politique, qui exige davantage de participation des gouvernés à l'élaboration des décisions les concernant directement dans leur vie quotidienne, cette participation passant par la mise en place de débats argumentés entre euxmêmes et les représentants des pouvoirs publics aux échelons locaux, ce qu'on appelle souvent la « démocratie de proximité ». Cet aspect est au centre de l'actualité dans un certain nombre de pays comme la France et le Canada ou, avec des variations considérables de signification, dans des pays pourtant apparemment très différents comme le Brésil ; il est caractéristique de l'évolution des démocraties occidentales, en gros depuis la fin des rêves utopistes nés dans les années 1966-1968, donc pour faire court à partir de 1975-1976. Le second aspect - apparu au centre de l'actualité politique aux États-Unis à peu près en même temps (début des années 80 ), mais de façon foncièrement indépendante, et qui réapparaît périodiquement depuis

\footnotetext{
5 Pour un tel traitement, je me permets de renvoyer à une version plus développée de cet article, disponible sur le site du département de sociologie de l'Université de Provence Aix-Marseille I (http://www.up.univ-mrs.fr/wsocio/textes/equipe/equipe.htm) (« La crise de légitimité des pouvoirs démocratiques contemporains, l'usage de la rhétorique et la valeur de l'argumentation. Crises et anti-crises rhétoriques »). Quelques comparaisons y sont également esquissées avec d'autres variétés de populisme caractéristique de l'époque plus immédiatement contemporaine (du début des années 80 et la montée des fondamentalismes religieux).
} 
l'instauration du parlementarisme aux XVIle et XVIII siècles - est nettement moins visible dans les débats, mais il est, à mon avis, plus profond. En effet, il correspond à une remise en cause chronique des dérives, elles-mêmes régulières et pour ainsi dire presque fatales, du pouvoir parlementaire des sociétés démocratiques, le débat argumenté qui devrait avoir lieu au sein des Parlements tendant périodiquement à faire place à de simples stratégies de partis, voire à de vulgaires marchandages, sapant en conséquence la nature même du pouvoir démocratique supposé rechercher rationnellement le bien public. Les deux crises anti-rhétoriques, quoique indépendantes, sont loin d'être sans rapport, car si les représentants du peuple visaient effectivement toujours rationnellement le bien public, la légitimité de leurs décisions serait certainement moins sujette à caution.

\section{Une anti-crise rhétorique caractéristique des démocraties actuelles : la valorisation des débats de proximité argumentés}

Examinons, pour commencer, un trait caractéristique tout récent de démocraties comme celles de la France et du Canada, des États-Unis et de l'Allemagne, et même du Brésil. II s'agit de l'émergence d'une démocratie plus « débattante » qu'auparavant - qu'on appelle souvent quoique assez abusivement « délibérative » - développant les débats entre citoyens eux-mêmes (voire entre gouvernés en général, puisqu'il n'est pas toujours nécessaire d'avoir la nationalité du pays concerné pour y participer). Dans ces débats, notamment dans certains spécialement organisés à cette fin, le recours à l'argumentation, au sens précédemment défini, est considéré comme une exigence et le recours à la mauvaise rhétorique, en certains cas, presque explicitement banni. Le Canada - plus précisément le Québec est souvent donné comme un exemple de démocratie pionnière en ce domaine, mais il s'agit peut-être d'un effet de perspective propre aux pays francophones, car il existe aussi des réalisations du même genre au Danemark, en Australie et, plus encore aux États-Unis où la tradition semble parfois remonter au tout début de la vie politique de ce pays avec l'expérience des meetings publics dans les municipalités des petites villes à peine nées (Gastil, Keith, 2005) - mais réciproquement peut-être faut-il faire la part de l'histoire mythique des origines - ou au Brésil, notamment à Porto Alegre qui fait figure de ville pionnière au niveau de la gestion directe de toute une partie des problèmes municipaux, quoique il s'agisse d'une ville de plus d'un million d'habitants (Gret, Sintomer, 2005) ${ }^{6}$.

${ }^{6}$ Je remercie G. Yanoshevsky pour des références comparatives concernant des pratiques du même genre en Israël. Voir notamment Goffer (2002). 
Ces débats argumentés concernent toujours des problèmes dits de proximité, par exemple au niveau d'une municipalité (comme à Porto Alegre), voire à des niveaux encore inférieurs (comme les conseils de quartier en France), niveaux qui, le plus souvent, n'ont pas d'existence administrative mais une existence purement géographique. En effet, il s'agit souvent de débats sur des questions qui concernent les riverains de grands projets d'aménagement du territoire d'intérêt régional, national ou international, mais à fortes répercussions en termes de nuisances pour les riverains immédiats. Globalement, si l'on fait exception d'une possible tradition séculaire aux États-Unis, l'émergence de ces débats a eu lieu quelques années après les mouvements protestataires des années 1966-1968 aux États-Unis, en France, en Allemagne, en Italie, mouvements souvent d'origine étudiante, en tout cas à forte composante intellectuelle, et remettant en cause les fondements de la démocratie représentative au nom de principes inspirés de diverses formes de démocratie directe, l'idée d'autogestion étant probablement la plus élaborée d'entre elles. Avec l'effondrement progressif de l'utopie communiste, ces mouvements protestataires se sont relativement vite essoufflés comme forces virtuellement révolutionnaires. En revanche, ils ont dynamisé des mouvements d'actions collectives d'une nature nouvelle puisque le mouvement ouvrier n'en était généralement plus le noyau, mais que celui-ci était constitué de groupes minoritaires divers (Habermas, 1987 ; Dubet, 1993 ; Touraine, 1993).

Ces mêmes mouvements ont aussi généré, en réaction, des réponses spécifiques des démocraties représentatives, non pas dans le sens d'une remise en cause de celles-ci, mais dans le sens de leur aménagement. C'est ainsi, en France, sous la Présidence de Valéry Giscard d'Estaing, dans les années 75, qu'est apparue l'idée d'une société modernisée dans son mode de mise en œuvre des décisions de l'exécutif (ce qu'on appelle maintenant la « gouvernance »), afin de trouver des moyens de restaurer l'autorité politique régulièrement mise à mal. Cependant, on date souvent des débuts du premier septennat de François Mitterrand, notamment de la loi Bouchardeau ${ }^{7}$ sur la démocratisation des enquêtes publiques et la protection de l'environnement, les vrais débuts de la démocratie de proximité. Ensuite, si l'on se concentre sur le cas de la France, les gouvernements de gauche (circulaire Bianco 1992) ${ }^{8}$ comme de droite

\footnotetext{
7 La loi Bouchardeau a été votée en 1983 à l'initiative d'H. Bouchardeau, ministre de I'Environnement, ancienne secrétaire nationale du Parti socialiste unifié (PSU), connu pour ses idées autogestionnaires.

8 J.-L. Bianco (membre du Parti socialiste), alors ministre de l'Équipement, du Logement et des Transports du gouvernement Bérégovoy, émit une circulaire le 15 décembre 1992 , dite « circulaire Bianco » pour organiser en pleins remous sociaux un débat destiné à désamorcer les conflits engendrés par la mise en place de la construction de la ligne ferroviaire duTGV Sud-Est.
} 
(loi Barnier 1995)9 ont œuvré dans le sens des mêmes aménagements en direction d'une participation accrue des riverains aux consultations, sinon aux décisions.

De quoi s'agit-il donc dans ce contexte ? Pas du tout d'une crise rhétorique au sens d'une critique de la valeur de l'argumentation au profit de la seule mauvaise rhétorique, mais de l'inverse. La crise, en effet, est politique. II s'agit d'une crique de l'autorité politique ou, en d'autres termes, une crise de la reconnaissance de la légitimité des décisions politiques de caractère pourtant légal (au sens banal comme au sens plus technique de MaxWeber), peut-être elle-même liée à une crise de représentativité des représentants du peuple, devenus impuissants à se faire les porte-parole des préoccupations de leurs administrés. Le but des réformes est de restaurer cette légitimité pour que, conformément aux lois en vigueur, les décisions prises par l'État soient appliquées sans heurts, en instaurant des procédures de décision qui recourent davantage à la justification argumentée des décisions et font place, symétriquement, aux contestations des riverains ou des gouvernés au niveau local, du moment que ces contestations sont argumentées. La valorisation de l'argumentation, au sens d'une procédure logiquement et éthiquement rationnelle tout en étant efficace dans son pouvoir de persuasion, est exactement ce qu'on a défini plus haut comme anti-crise rhétorique.

Cette valorisation des débats argumentés a des fondements de diverses factures. Les plus évidents, ceux qui ont probablement été effectifs au niveau des ministères successifs, sont fort prosaïques. II s'agit simplement d'accroître l'efficacité des prises de décision au niveau national par des procédures adaptées à ce qu'on pourrait appeler un double mouvement convergent: d'un côté, de démocratisation toujours plus grand de la gestion des affaires politiques; de l'autre, de rationalisation générale de la culture ambiante conduisant à tendre à demander raison de tout. Mais il y a d'autres justifications, dont il est difficile de préciser l'impact effectif sur le processus de généralisation de ces procédures de débats publics argumentés.

En effet, il faut se garder de l'illusion rétrospective. Par exemple, ce n'est pas parce la version anglaise d'un article de Bernard Manin, paru initialement en 1985 en français dans Le Débat a eu un rôle important

\footnotetext{
9 M. Barnier est l'auteur d'une loi, parue au JO le 2 février 1995 relative à la participation du public et des associations en matière d'environnement, loi qui offre un cadre juridique aux débats entre les promoteurs de projets d'aménagement ou d'équipement ayant une incidence importante sur l'environnement ou l'aménagement du territoire et les riverains du projet en question.
} 
au niveau international ${ }^{10}$ dans l'élaboration philosophique de l'idée de démocratie délibérative - Jürgen Habermas (1992) lui-même, pourtant un des pionniers en ce domaine, le cite dans la préface à la réédition de sa thèse sur l'espace public -, que la version initiale a eu un rôle analogue au niveau des législations nationales dans l'élaboration juridique des procédures de débat public, même en France. Cet article a en effet été redécouvert au tout début des années 2000, et dans le contexte plutôt militant des partisans d'expériences comme celle de Porto Alegre (Gret, Sintomer, 2005). À noter que Bernard Manin lui-même s'étonnait de I'histoire curieuse de la diffusion et de l'impact de son article (Blondiaux, 2002).

Quoi qu'il en soit, la source de justification la plus élaborée de ces débats se trouve chez Jürgen Habermas, non sans ambiguïté d'ailleurs, car la position de ce dernier a sensiblement évolué entre la Théorie de l'agir communicationnel (1981) et Droit et démocratie (1992). L'idée habermassienne essentielle sur ces questions va bien au-delà de la politique du débat argumenté puisque l'argumentation est supposée jouer un rôle fondamental dans l'éthique même, dans la mesure où le principe kantien d'universalisation - pouvoir ériger les maximes qui guident nos actions particulières en principes universels d'action - doit être complété par un principe de discussion (argumentée), la formulation de tels principes pouvant être sujette à désaccord (Habermas, 1983). Mais Jürgen Habermas reste vague sur la façon dont cette éthique de la discussion peut être mise en œuvre en politique. Dans la Théorie de l'agir communicationnel, il s'agit essentiellement de forums informels et l'application du principe d'universalisation à la politique - qui pourrait signifier qu'il faut rechercher le bien public - reste indéterminée. En outre, il est implicitement question de débats en quelque sorte horizontaux, c'est-à-dire entre participants aux mouvements de protestation et pas du tout, du moins de façon explicite, de débats en quelque sorte verticaux, entre représentants de l'État et gouvernés, par exemple entre maître d'œuvre sollicité par l'État et riverains d'un grand projet d'aménagement du territoire, comme c'est le cas dans les procédures de débat public évoquées ici. On parle souvent à ce propos de politique délibérative ou de démocratie délibérative, mais à la faveur d'un glissement de sens, l'expression étant réservée par Jürgen Habermas dans Droit et démocratie, conformément à l'usage américain de deliberative democracy depuis Joseph Bessette (1980), aux débats au sein du Parlement auxquels sera consacrée la seconde partie de cet article.

${ }^{10}$ L'article est paru en anglais en 1987 dans la revue Political Theory. 
En effet, qui dit délibération dit en principe, dans la langue philosophique classique comme dans la langue juridique moderne,pouvoir de « décision », au point qu'avoir voix délibérative dans une assemblée, par opposition au fait de ne disposer que d'une voix consultative, signifie purement et simplement avoir le pouvoir de décider. Or, les débats démocratiques de proximité en dehors des conseils de quartier, qui concernent des domaines d'application extrêmement limités, n'ont précisément pas ce pouvoir de décider. La confusion est un peu induite aussi par l'opposition de Jon Elster (1986), très utile quand elle est appliquée aux seuls Parlements et sur laquelle je reviendrai, entre argumenter, négocier et voter comme trois procédures types de décision en elles-mêmes indépendantes. Car l'argumentation dont il s'agit est celle qui aboutit à une décision ; il s'agit donc plutôt, dans ce cas-là, d'une délibération.

Toutes les procédures de débat de proximité ne valorisent pas autant l'argumentation. Ainsi la procédure dite de débat public en France, introduite en 1995 et perfectionnée en 2002'", la valorise-t-elle beaucoup plus que les procédures de consultation des citoyens au Québec et plus aussi que les «conférences de citoyens 》, répandues au Danemark. Cette procédure française est requise dès lors qu'un grand projet d'aménagement du territoire est projeté : elle conduit à organiser des débats entre maître d'œuvre et riverains, sous le contrôle d'une commission particulière dont les membres sont nommés par une commission nationale dont les membres le sont eux-mêmes par le gouvernement. Cette commission particulière a pour unique mission d'organiser les débats et de veiller à leur bonne tenue. Elle ne représente donc pas le point de vue du gouvernement et pas davantage celui du maitre d'œuvre; au contraire, elle exerce une fonction d'arbitre entre celui-ci et les riverains. C'est cette position tierce qui lui permet de garantir la qualité formelle ou procédurale - notamment argumentative - du débat.

En outre, toutes les expériences de débat public ne satisfont pas au même degré l'exigence d'argumentation dans le débat parce que la commission particulière, et notamment son président, ne se font pas forcément la même idée de ce que doit être leur rôle, la loi restant assez vague sur ce point. On s'accorde souvent à voir dans le deuxième débat de type loi Barnier qui a eu lieu, en l'occurrence en 1998 en Provence-Côte d'Azur,

\footnotetext{
"Le décret d'application du 10 mai 1996 de la loi Barnier de 1995 (voir ci-dessus), a créé une Commission nationale du débat public - CNDP - (dont les attributions sont renforcées par aménagée par la loi du 22 Février 2002 relative à la démocratie de proximité,). La CNDP nomme une commission régionale du débat public chargée d'organiser un débat dans les cas prévus par la loi.
} 
une réalisation particulièrement exemplaire, en raison notamment de la conception exigeante que s'est faite de son rôle son président, Gérard Porcell (Bouvier, 2007b).

Cependant, au fur et à mesure que se multiplient ces débats, augmente aussi la contestation de la procédure. Cette contestation a deux sources essentielles: la première, plutôt marginale en France et qui vient notamment des féministes néo-marxistes américaines, consiste à contester le principe même de la discussion argumentée, au motif qu'elle serait antidémocratique puisque tout le monde n'a pas le même talent rhétorique ni la même aisance à s'exprimer en public ; la seconde, majoritaire dans le contexte français, consiste à contester le principe du débat public au motif que la décision soit serait déjà prise, soit ne tiendrait pas compte ou peu du débat, ce qui revient à peu près au même. La première contestation relèverait, si elle s'amplifiait, de ce que j'ai appelé une crise rhétorique. La seconde manifeste plutôt une réapparition de la crise d'autorité politique que ce genre de procédure est pourtant supposé résoudre. En effet, cette crise de nature politique consiste à remettre implicitement en cause le principe même de la démocratie représentative en considérant que les participants au débat devraient aussi participer à la décision, ce qui n'est pas possible sauf à donner le pouvoir aux intérêts locaux, même quand des intérêts plus larges sont en jeu. Pour prendre un exemple: il n'était pas raisonnable de laisser aux seuls habitants de la vallée du Rhône - même s'ils ont à subir seuls les inconvénients de l'installation de la voie ferrée du TGV - le pouvoir de décider s'il fallait ou non installer cette voie, puisque les utilisateurs n'en sont pas seulement les riverains. En revanche, ce qui était possible, c'est que le contenu des débats remonte effectivement vers les décideurs, ce qui est le cas depuis la loi de 2002 mais ne l'était pas dans la loi de 1995 (et a fortiori avant); autrement dit, les participants au débat n'avaient même pas voix consultative. En tout état de cause, on ne confondra pas cette crise de l'autorité politique avec la crise rhétorique mentionnée.

\section{Une seconde anti-crise rhétorique caractéristique des démocraties actuelles : la revalorisation des délibérations parlementaires}

La vague de l'idée de démocratie délibérative, qui met au premier plan l'idée de débats politiques argumentés, tend le plus souvent à confondre deux niveaux de débats politiques possibles (Bouvier, 2007a) - en partie en raison d'une certaine ambiguïté chez Jürgen Habermas lui-même, au moins dans ses premiers textes sur le sujet - de sorte qu'on tend à confondre ce qu'il faut pourtant appeler deux anti-crises rhétoriques de 
nature différente. Comme on l'a vu, les débats argumentés peuvent avoir lieu entre les citoyens ordinaires et même entre les simples résidents d'un pays, qu'ils soient ou non citoyens de ce pays, plus précisément qu'ils aient ou non la nationalité du pays en question, a fortiori qu'ils soient ou non possesseurs de droits civiques tel le droit de voter. Ces débats peuvent être informels, comme cela arrive dans le cadre de mouvements protestataires encore inorganisés - et c'est à eux que Jürgen Habermas semblait penser à l'époque de la Théorie de l'agir communicationnel ( I98I). Ils peuvent aussi être formalisés et organisés par les gouvernements en exercice (voir supra). Mais les débats argumentés en démocratie peuvent également avoir lieu entre les représentants des citoyens, au niveau des Parlements, par exemple en France à l'Assemblée nationale et au Sénat et, aux États-Unis, au niveau du Congrès. Or, comme on l'a mentionné en introduction en faisant allusion à la République de Weimar, on peut aussi parler de crise rhétorique à ce niveau proprement parlementaire.

Il convient ici de se projeter dans les États-Unis de la fin des années 70, car c'est très exactement dans ce contexte - et non dans celui, germanique, qui a nourri la pensée de Jürgen Habermas - qu'est apparue la notion de « deliberative democracy ». En effet, tous les spécialistes s'accordent à reconnaître que l'expression est née sous la plume de Joseph Bessette (1980). Or, le contexte était celui d'une mise en cause des pratiques du Congrès américain, auquel on reprochait de ne plus du tout délibérer, plus précisément de ne plus être le théâtre d'échanges argumentés entre les représentants du peuple mais de donner lieu, à la place, soit à de simples votes de partis dont la position était déterminée en dehors du Congrès et avant les débats ou plutôt les pseudo-débats, soit à des votes entérinant des marchandages plus individuels entre membres du Congrès. La crise que stigmatisait Joseph Bessette était bien une crise rhétorique, au sens défini, puisque la valeur de l'argumentation était manifestement déniée de facto au sein du Parlement au profit de ce qui relève de la simple rhétorique visant l'efficacité, ce qui est assez caractéristique des procédures de marchandage. Mais la réaction de Joseph Bessette, qui a eu un large écho car elle exprimait un sentiment très largement répandu chez les analystes politiques, a également eu un impact considérable au niveau tant de la sociologie politique que de la philosophie politique et du droit constitutionnaliste lui-même. Autrement dit, le diagnostic de crise rhétorique, modulé ensuite par ce Joseph Bessette (1994), a provoqué une anti-crise rhétorique, en l'espèce une réaction politicojuridique, mais aussi philosophique, en faveur de la revalorisation de l'argumentation au sein du Congrès. Au demeurant, la réaction de Joseph Bessette n'est pas sans présenter quelque analogie avec des réactions du même ordre en Europe, mais qui ont eu moins d'échos et dont on peut dire qu'elles sont chroniques au sein des institutions parlementaires. 
Toutefois, la crise rhétorique américaine des années 70 avait des fondements probablement plus profonds que d'autres crises du même type parce qu'elle est liée au développement d'une école de sociologie politique, l'école de Rochester, et d'un courant d'analyse dite « économique » de la démocratie (dont le principal représentant est Anthony Downs) et à leur impact sur la politique des grands partis. Dans Economic Analysis of Democracy (1957), Anthony Downs appliquait la théorie dite du choix rationnel au fondement de la démocratie en montrant qu'on comprenait mieux le fonctionnement de celle-ci si l'on supposait les hommes politiques guidés par la volonté de leur élection ou de leur réélection que par la recherche du bien public ou de quelque valeur que ce soit, ces valeurs étant choisies plutôt en fonction des électeurs potentiels disponibles que des convictions des candidats. Le point de vue d'Anthony Downs n'était pas normatif, mais il semble bien qu'il ait été parfois pris comme tel par un certain nombre d'hommes politiques cyniques qui y ont vu un moyen de rationaliser leur pratique par des techniques de marketing.

Jon Elster (1986, I994, 1998) a joué un rôle majeur dans la thématisation de cette crise et - bien au-delà - dans l'explicitation des difficultés que rencontrent toutes les procédures de choix collectif,mais indépendamment d'Anthony Downs et plutôt en réaction contre lui, en opposant trois grands types de décision en matière collective, qu'on peut rencontrer sous des formes hybrides mais aussi à l'état pur : voter, négocier (ou marchander, bargaining) et argumenter. Jon Elster participe à la vague de l'anti-crise rhétorique puisque, sans ambiguïté, il valorise l'argumentation. II donne à celle-ci un sens particulièrement fort, du reste, puisque argumenter au sens où il entend ce terme, c'est chercher à persuader rationnellement non seulement au sens de l'efficacité, de la logique et de l'éthique, mais plus précisément afin de viser cette forme de valeur particulière qu'est le bien public. De fait, l'argumentation telle que la conçoit Jon Elster est proche de la délibération au sens d'Aristote.

Lorsque Jon Elster élabore cette distinction en 1986, il vient de rencontrer la pensée de Jürgen Habermas qui a publié les Notes programmatiques pour fonder en raison une Éthique de la discussion peu auparavant (1983) et qui ne se préoccupe pas encore directement de politique délibérative. Or, cette rencontre ne se comprend que sur le fond des débats proprement américains: Jon Elster ne cherche nullement à développer des forums informels de contestataires, ni même à prôner l'organisation par l'État de débats de proximité pour restaurer l'autorité politique des gouvernements en régime de démocratie représentative en aménageant cette dernière. Cette crise de légitimité des États démocratiques modernes qui rend délicate l'exécution de leurs décisions lui est étrangère. II s'intéresse seulement à la crise des processus mêmes de décisions de ces derniers. Et l'anti-crise rhétorique dont il se trouve être, de facto, un représentant 
est donc d'une tout autre nature que l'anti-crise rhétorique évoquée précédemment et qui donne lieu au développement de la démocratie de proximité en France, au Canada et aux États-Unis mêmes.

\section{Conclusion}

Le propos de Joseph Bessette était de revaloriser l'idée de délibération; or celle-ci, selon lui, est caractéristique de l'idée même de démocratie représentative telle qu'elle avait été formulée par les pères de la Constitution américaine. C'est ce que confirment largement les historiens du Droit américain. Et c'est sur cet aspect que je conclurai cette analyse parce que les débats des Federalist Papers qui ont conduit à la Constitution de 1787 (toujours en vigueur avec addition d'amendements successifs), illustrent le fait que l'idée de démocratie représentative (non pas celle de démocratie participative), est concomitante, tout particulièrement dans sa variante américaine par opposition à la variante française, d'une anti-crise rhétorique, c'est-à-dire d'une valorisation de l'argumentation. Dans les débats qui ont opposé Thomas Jefferson et James Madison sur fond d'un large accord sur l'essentiel, le second avançait comme argument clé en faveur de la démocratie représentative l'idée que la délibération, donc l'échange d'arguments, n'était possible qu'entre les représentants du peuple, non entre les membres du peuple lui-même dès lors que, par délibération, on entendait échanges argumentés en vue du bien public précédant une décision.

Les arguments de James Madison peuvent aujourd'hui paraitre soit obsolètes, soit biaisés par les préjugés, celui-ci invoquant l'absence d'éducation du peuple et sa propension à se laisser guider par les passions, alors que l'éducation a beaucoup progressé et que la soumission aux passions n'est probablement pas l'apanage d'une classe sociale particulière. Mais, d'un autre côté, on sait bien, d'une part, qu'un faible niveau d'éducation rend délicat l'exercice de l'argumentation - les néo-marxistes américains sont là pour le répéter -, d'autre part, que la démocratie directe ne peut, pour des raisons purement matérielles, prendre une forme argumentative. Dans les référendums, il peut y avoir énoncé d'arguments dans la presse ou dans les conversations précédant le vote, mais pas véritablement échange d'arguments entre toutes les parties concernées.Au demeurant,l'expérience de démocratie de proximité de la municipalité de Porto Alegre manifeste une nouvelle fois qu'à partir d'un certain nombre de participants, on ne peut se passer de représentants. Marion Gret et Yves Sintomer (2005) montrent bien comment les habitants de Porto Alegre retrouvent, dès lors, les problèmes mêmes qui étaient ceux d'Emmanuel-Joseph Siéyès pendant la Révolution française, comme l'exigence de ne pas donner de mandats impératifs aux représentants - au risque d'être accusé de ne plus être les porte-parole des représentés - si l'on veut que les représentants soient à 
même de modifier leur position au cours de la délibération, en vue même de parvenir au bien public.

Si les débats sur la Constitution américaine de 1787 sont plus importants pour ce qui nous intéresse que ceux de la Constituante française de 1789179|, c'est que la dimension d'anti-crise rhétorique y apparaît de manière beaucoup plus nette. En effet, ce sont les constitutionnalistes américains, spécialement James Madison, qui ont mis au premier plan l'exigence de délibérer, donc d'argumenter. Alors que les constitutionnalistes français restaient davantage marqués par l'idéal rousseauiste d'une harmonie des volontés, laquelle pourrait être obtenue, à la manière de Rousseau, par l'union des cœurs plus que par celle des raisons (Manin, 1985; Habermas, 1992). Pour résumer : la période contemporaine ne me semble pas marquée - au moins pour ce qui est au cœur des démocraties occidentales - par une crise rhétorique, quoiqu'il existe une double crise politique, au niveau des prises de décision parlementaires, au niveau de la légitimité de ces décisions. Mais l'époque invite plutôt à l'optimisme au niveau de la reconnaissance de la valeur de l'argumentation puisqu'on peut parler symétriquement d'une double anti-crise rhétorique.

\section{Références}

Bessette J., 1980, « Deliberative Democracy : The Majority Principle in Republican Government », pp. 102-1 I6, in : Goldwin R., Schambra W., eds, How Democratic is the Constitution?,Washington, American Entreprise Institute.

- 1994, The Mild Voice of Reason: Deliberative Democracy and American National Government, Chicago, Chicago University Press.

Blondiaux L., 2002, « Entretien avec Bernard Manin : l'idée de démocratie délibérative dans la science politique contemporaine. Introduction, généalogie et éléments critiques », Politix, vol. 15, 57, pp. 37-52.

Bouvier A., 2007a, «Démocratie délibérative, démocratie débattante, démocratie participative », Revue européennes des sciences sociales, « Démocratie participative, démocratie délibérative? »,T. XLV, I36, pp. 5-34.

- 2007b, «La dialectique des relations de confiance et d'autorité au sein de la démocratie dite "participative" et "délibérative". Un exemple paradigmatique : le débat public "loi Barnier" en Provence-Côte d'Azur (1997-8) concernant le projet d'une construction de ligne électrique à Très HauteTension », Revue européennes des sciences sociales, T. XLV, I 36, pp. I8I-230.

— 2007c, « La crise de légitimité des pouvoirs démocratiques contemporains, l'usage de la rhétorique et la valeur de l'argumentation. Crises et anti-crises rhétoriques », site http://wnw.up.univ-mrs.fr/wsocio/textes/equipe/equipe.htm

Douay Fr., 2007, «Révolutions et crises de la rhétorique. Le cas de la révolution française », Colloque international en argumentation, Crises rhétoriques et crises démocratiques, Bruxelles, Université libre de Bruxelles, I0-I I mai. 
Downs A., 1957, An Economic theory of democracy, New York, Harper.

Dubet Fr., 1993, « Les nouveaux mouvements sociaux », pp. 61-69, in : Chazel F., dir., Action collective et mouvements sociaux, Paris, Presses universitaires de France..

Elster J., 1986, «The market and the forum », in : Elster J., Hylland A., Foundations of social choice theory, Cambridge, C.U.P., repris pp. 3-33, in : Bohman J., Rehg W., eds, 1997, Deliberative Democracy. Essays on Reason and Politics, Cambridge, Mass., MIT Press.

- 1994, «Argumenter et négocier dans deux assemblées constituantes », Revue française de science politique, vol. 44, 2, pp. I87-256.

— dir., 1998, Deliberative Democracy, Cambridge, C.U.P.

Gastil J., Keith, W., 2005, « A Nation that (sometimes) Likes to Talk. A Brief History of Public Deliberation in the United States », pp. 3-19, in : Gastil J., Levine P., eds, Handbook of Deliberative Democracy : Strategies for Effective Civic Engagement in the twenty-first century, San Francisco, Jossey Bass.

Gastil J., Levine, P., eds, 2005, Handbook of Deliberative Democracy : Strategies for Effective Civic Engagement in the twenty-first century, San Francisco, Jossey Bass.

Goffer R., 2002, « Le droit de parole donné aux citoyens » (en hébreu), Gilyonot, 21.

Gret M., Sintomer Y., 2005, Porto Alegre. L'espoir d'une autre démocratie, Paris, Éd. La Découverte.

Habermas J., 1962, « Préface à l'édition de 1990 » de L'espace public.Archéologie de la publicité comme dimension constitutive de la société bourgeoise, trad. de l'allemand par M. de Launay, Paris, Payot, 1992.

— 198I, Théorie de l'agir communicationnel, trad. de l'allemand par Ferry J.-M., Paris, Fayard, 1987.

- 1983, «Notes Programmatiques pour fonder en raison une Éthique de la discussion », pp. 63-130, in : Habermas J., dir., Morale et communication. Conscience et activité communicationnelle, trad. de l'allemand par Chr. Bouchindhomme, Paris, Éd. du Cerf, 1996.

— 1992, Droit et démocratie, trad. de l'allemand par R. Rochlitz, Chr. Bouchindhomme, Paris, Gallimard, 1997.

Manin B., 1985, «Volonté générale ou délibération? Esquisse d'une théorie de la délibération publique », Le Débat, 33, pp. 72-93.

Mill J. St., | 843, Système de logique déductive et inductive, Liège, Mardaga, 1988.

Touraine A, 1993, « Découvrir les mouvements sociaux », pp. 17-36, in : Chazel F., dir., Action collective et mouvements sociaux, Paris, Presses universitaires de France. 